\title{
Comparison of Central Subfoveal Choroidal Thickness in Diabetic Patients with and without Diabetic Retinopathy
}

\author{
Muhammad Ali Haider ${ }^{1}$, Uzma Sattar ${ }^{2}$, Muhammad Amjad ${ }^{3}$ \\ ${ }^{1,2}$ Rahbar Medical \& Dental College, Punjab Rangers Teaching Hospital, Lahore \\ ${ }^{3}$ Huddersfield Royal infirmary, UK, Calderdale Royal Hospital, Halifax, UK
}

\begin{abstract}
Purpose: To compare the Central subfoveal choroidal thickness (CCT) in diabetic patients with and without diabetic retinopathy.

Study Design: Cross sectional observational study.

Place and Duration of Study: Al-Ehsan Welfare Eye Hospital, Lahore, from June 2019 to May 2020.

Methods: One hundred and twenty patients with type II diabetes were included with a mean age of $57 \pm 0.9$. Patients were divided into two groups (60 in each group) based on presence or absence of diabetic retinopathy after ophthalmic examination. Group A comprised of patients showing signs of retinopathy and group B with no signs of diabetic retinopathy. After detailed ophthalmic examination, spectral-domain OCT was performed for the measurement of central subfoveal choroidal thickness. The differences in measurements were analyzed and measured by using SPSS version 22.
\end{abstract}

Results: Out of 120 patients, 69 (57.5\%) were males and 51 were females (42.5\%). Mean age of patients was $58.8 \pm 10$ years with minimum 28 years and maximum 94 years. Central choroidal thickness in group A showed a mean value of $239 \pm 41 \mu \mathrm{m}$ with standard error of mean 3.76. While diabetic patients having no signs of diabetic retinopathy (Group B) showed mean subfoveal choroidal thickness of $240 \pm 42 \mu \mathrm{m}$ with standard error of mean 3.89. The difference in central subfoveal choroidal thickness in both groups was 1.337 which is statistically insignificant with $p$ value of 0.250 .

Conclusion: Central choroidal thickness amongst diabetic patients with and without signs of retinopathy does not have any significant changes.

Key Words: Choroid, Diabetic retinopathy, Optical Coherence Tomography.

How to Cite this Article: Haider MA, Sattar U, Amjad M. Comparison of Central Subfoveal Choroidal Thickness in Diabetic Patients with and without Diabetic Retinopathy. Pak J Ophthalmol. 2021, 37 (3): 283-288.

Doi: $10.36351 /$ pjo.v37i3.1207

\section{INTRODUCTION}

Diabetes mellitus due to chronic hyperglycemia can stimulate ocular complications especially micro

Correspondence: Muhammad Ali Haider

Rahbar Medical \& Dental College, Lahore

Email: alihaider_189@yahoo.com.

Received: January 17, 2021

Accepted: April 28, 2021 vascular abnormalities including retinal and choroidal vasculature. These ocular micro vascular abnormalities affect the quality of patient's life by influencing vision. ${ }^{1}$

Diabetic retinopathy leads to significant capillary drop-out that in turn causes non-perfusion of retinal tissue. ${ }^{2}$ abnormal choroidal performance due to hyperglycemia include luminal narrowing, capillary drop-out and choroidal neovascularization. The most important parameter for analyzing choroidal 
vasculature abnormality is choroidal thickness measurement. Retinal pigment epithelium and the outer layers of retina derive their nutrition from the choroid so disturbed hemodynamics of choroidal vasculature can affect their thickness. ${ }^{3}$

Recently, many studies have been published on using choroidal thickness changes as an indicator of early retinal and choroidal vascular abnormalities. ${ }^{4}$ Previously it was thought that diabetic retinopathy is a disease which primarily affects the retinal vasculature resulting in retinopathy but recent studies have highlighted that thinning of choroiocapillaris also occurs in diabetic patients..$^{5}$ Doppler flowmetry in early diabetic retinopathy has also shown decreased choroidal vessels perfusion. ${ }^{6}$ On scanning electron microscopy, eyes with long-standing diabetes show increased vascular tortuosity, dilation and narrowing, hyper cellularity, vascular loop and micro aneurysm formation, drop-out of choroiocapillaris and sinus-like structure formation between choroidal lobules. ${ }^{7,8}$

Blood flow through the choroid also appears to be reduced in subjects with diabetes, especially those with macular edema. In vivo evaluation of choroid using enhanced depth imaging has also shown various changes in choroidal structures in recent literature. It thus follows that, structural alterations in the choroid, if any, may contribute to the pathogenesis of diabetic retinopathy. ${ }^{9}$

The rationale of this study was to find out the central sub-foveal choroidal thickness in patients with diabetic retinopathy and compare with those without retinopathy. It may help in early detection of the microvascular changes before retinopathy actually sets in.

\section{METHODS}

This cross-sectional study was carried out at the retinal unit of Al-Ehsan eye hospital, Lahore after approval from the ethical committee. Total 120 patients were enrolled in this study. Patients, who were diagnosed with diabetic retinopathy and underwent OCT were included in group 'A'. A control group 'B' was created of diabetic patients without any signs of diabetic retinopathy. Consent, demographic profile and detailed history were recorded. All participants underwent recording of visual acuity, slit-lamp examination, intraocular pressure (IOP) measurement and dilated fundal examination. Diabetic retinopathy classification in group A was performed as per Early
Treatment Diabetic Retinopathy Study (ETDRS) criteria. In case where there was any doubt as to the stage of DR, fundus fluorescein angiography was performed.

Inclusion criteria for group A included a history of Type II diabetes mellitus, treated with either oral hypoglycemic agents or insulin and having non proliferative or proliferative diabetic retinopathy changes without diabetic macular edema. Exclusion criteria comprised of media opacities hampering fundal view and imaging, high myopia or hyperopia $(>-6$ or +6 diopters of refractive error), presence of diabetic macular edema, history of treatment with antiVEGF or pan-retinal photocoagulation, any associated retinal pathology and previous history of retinal surgery.

Optical coherence tomography scans were obtained by using NIDEK RS-300 Advance angioOCT Gamagori, Japan (Spectral domain OCT Optical Z: $7 \mu \mathrm{m}, \mathrm{X}-\mathrm{Y}: 20 \mu \mathrm{m}$, Digital Z: $4 \mu \mathrm{m}, \mathrm{X}-\mathrm{Y}: 3 \mu \mathrm{m}, \mathrm{X}$ : 3 to $12 \mathrm{~mm}$ (12 mm for line scan only), Y: 3 to $9 \mathrm{~mm}$, Z: $2.1 \mathrm{~mm}$, SLD, $880 \mathrm{~nm}$, Max. 53,000 A-scans / s, 1.6 $\mathrm{s}$ in regular mode). The scan used for imaging in this study was HD 1-line raster with enhanced depth imaging. The sclera-choroidal interface was drawn manually in the macular map X-Y (9 x 9mm [512 x 128]) scan. Choroidal thickness was measured between the hyper-reflective outer border of the retinal pigment epithelium (RPE) and the line of sclerachoroidal interface, and central thickness was measured as subfoveal distance in micrometer between the outer borders of retinal pigment epithelium to the line of sclera-choroidal interface.

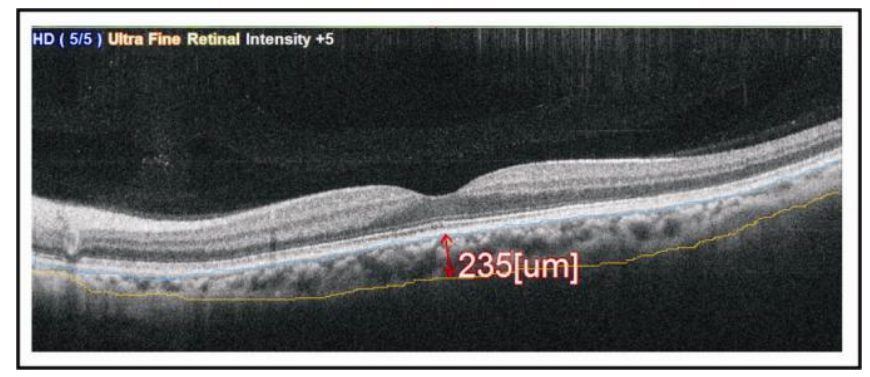

Figure 1: One line raster, HD OCT scan showing choroidal thickness.

Data was analyzed by using SPSS version 22. All the qualitative variables were analyzed through descriptive statistics. For quantitative variables, normality assumption was checked by using Shapiro- 
Wilk and Kolmogorov-Smirnov tests. Subfoveal choroidal thickness was compared in both groups of diabetics with and without retinopathy by using Levene's Test for Equality of Variances. A p value of $<0.01$ was considered significant.

\section{RESULTS}

Total 120 patients were enrolled in this study. Out of which $69(57.5 \%)$ were males and 51 were females $(42.5 \%)$. Mean age of patients was $58.8 \pm 10$ years with minimum of 28 years and maximum of 94 years. Central choroidal thickness in patients with diabetic retinopathy (Group A) showed a mean value of $239 \pm$ $41 \mu \mathrm{m}$ with standard error of mean 3.76. While diabetic patients having no any sign of diabetic retinopathy (Group B) showed mean subfoveal choroidal thickness of $240 \pm 42 \mu \mathrm{m}$ with standard error of mean 3.89. Normality test of central choroidal thickness with and without diabetic retinopathy showed a $\mathrm{p}$ value of more than 0.01 indicating statistically insignificant difference between the two groups (Table 1 and 2).

Table 1: Tests of Normality.

\begin{tabular}{|c|c|c|c|c|c|c|}
\hline & \multicolumn{3}{|c|}{ Kolmogorov-Smirnov ${ }^{\mathrm{a}}$} & \multicolumn{3}{|c|}{ Shapiro-Wilk } \\
\hline & Statistic & df & Sig. & Statistic & df & Sig. \\
\hline CCT with DR & .105 & 120 & .002 & .973 & 120 & .017 \\
\hline $\begin{array}{l}\text { CCT without } \\
\text { DR }\end{array}$ & .052 & 120 & $.200^{*}$ & .990 & 120 & .548 \\
\hline
\end{tabular}

*. This is a lower bound of the true significance.

a. Lilliefors Significance Correction

Table 2: Comparison of subfoveal Central Choroidal Thickness in Group A and Group B applying independent sample test.

Independent Samples Test

Levene's Test for
Equality of Variances

$\begin{array}{lll}95 \% \text { Confidence } & \\ \text { Sig. (2- Mean } \quad \text { Std. Error Interval of the }\end{array}$

F Sig. $\quad t \quad$ Df $\quad$ Sig. (2- Mean Std. Error Interval of the

\begin{tabular}{rllllllll} 
& F & Sig. & t & Df & $\begin{array}{l}\text { Sig. (2- } \\
\text { tailed) }\end{array}$ & $\begin{array}{l}\text { Mean } \\
\text { Difference }\end{array}$ & $\begin{array}{l}\text { Std. Error } \\
\text { Difference }\end{array}$ & $\begin{array}{l}\text { Interval of the } \\
\text { Difference } \\
\text { Lower }\end{array}$ \\
\hline Upper
\end{tabular}

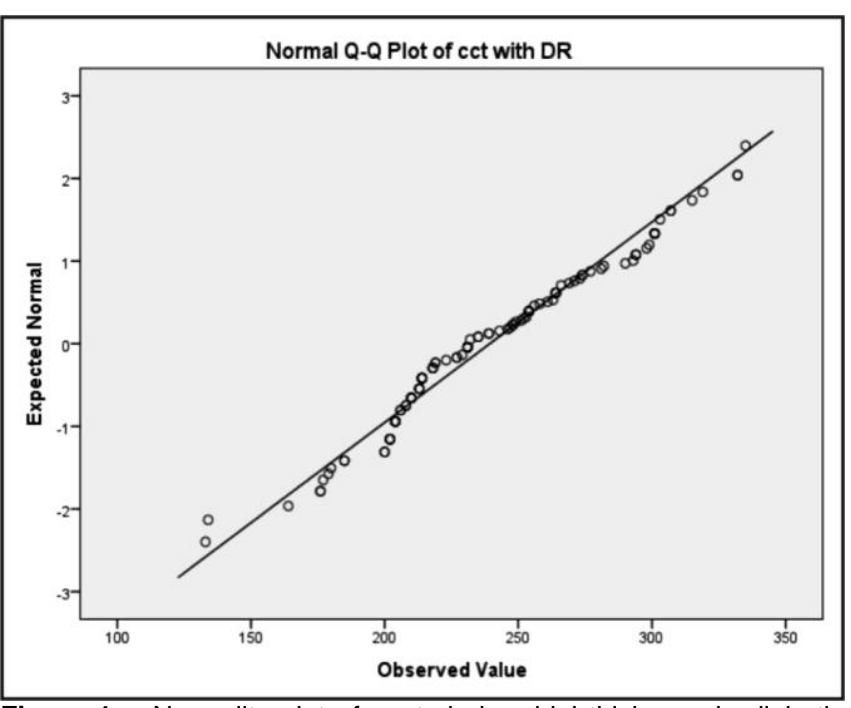

Figure 1a: Normality plot of central choroidal thickness in diabetic patients with diabetic retinopathy (Group A).

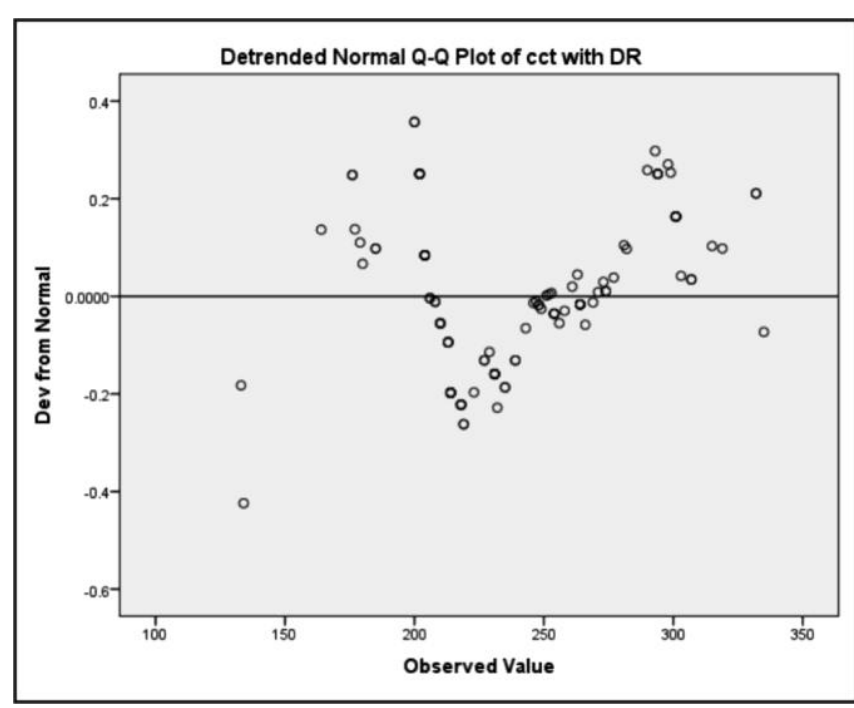

Figure 1b: Normality plot of central choroidal thickness in diabetic patients with diabetic retinopathy (Group A). 


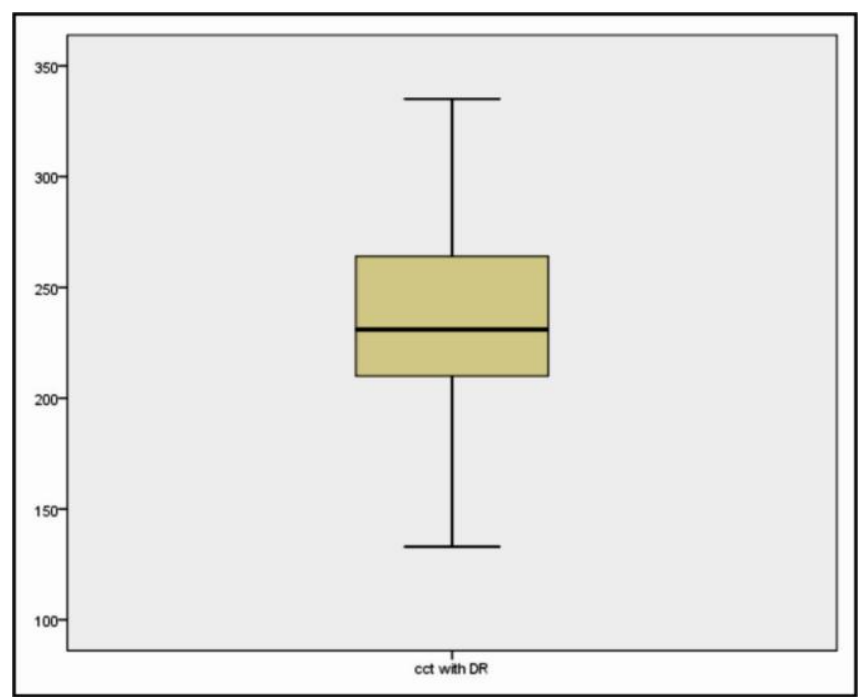

Figure 1c: Normality plot of central choroidal thickness in diabetic patients with diabetic retinopathy (Group A).

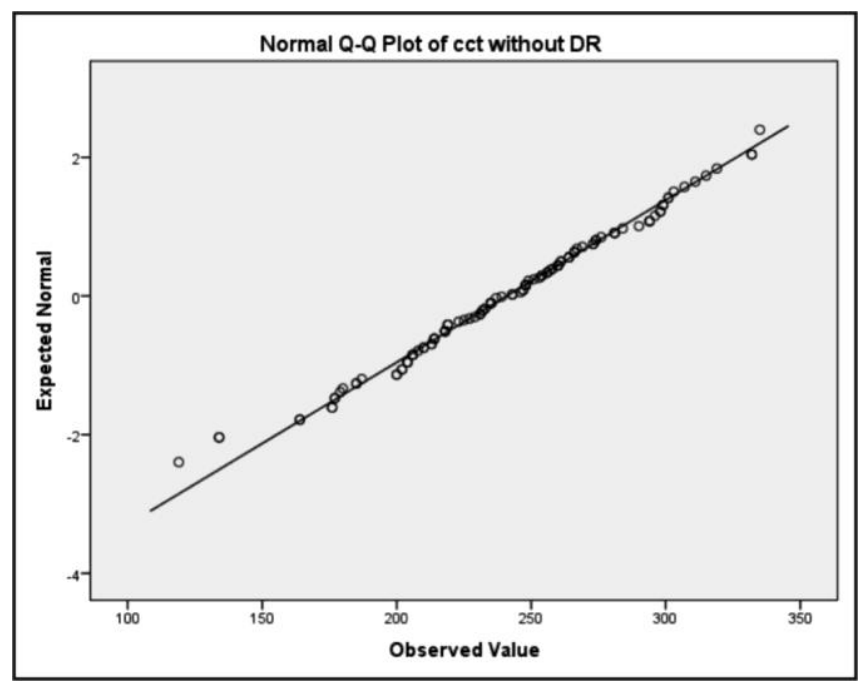

Figure 2a: Normality plot of central choroidal thickness in diabetic patients without diabetic retinopathy (Group B).

\section{DISCUSSION}

Enhanced depth imaging optical coherence tomography has revolutionized choroidal thickness measurements, which are useful in aiding diagnosis of diabetic eye diseases. Choroidal thickness can predict the response to anti angiogenic agents in retinal pathologies including diabetic retinopathy. ${ }^{10}$ While analysis of data on choroidal thickness in subjects with diabetes have been published, recent studies have reported conflicting results. Rewbury et al. published a systematic review of articles on sub-foveal choroidal thickness in diabetic patients. As per their results, severity of diabetic retinopathy correlated with

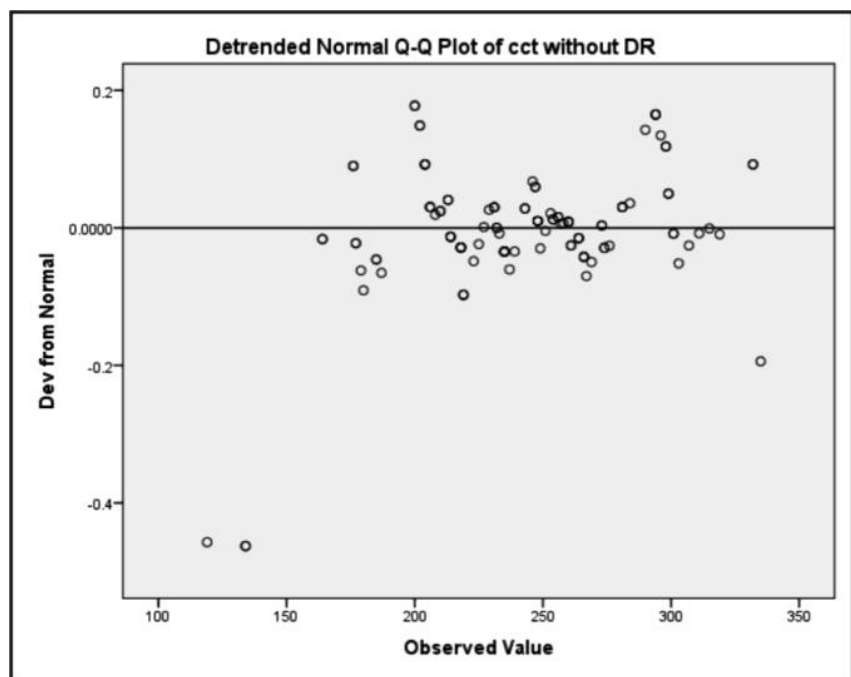

Figure 2b: Normality plot of central choroidal thickness in diabetic patients without diabetic retinopathy (Group B).

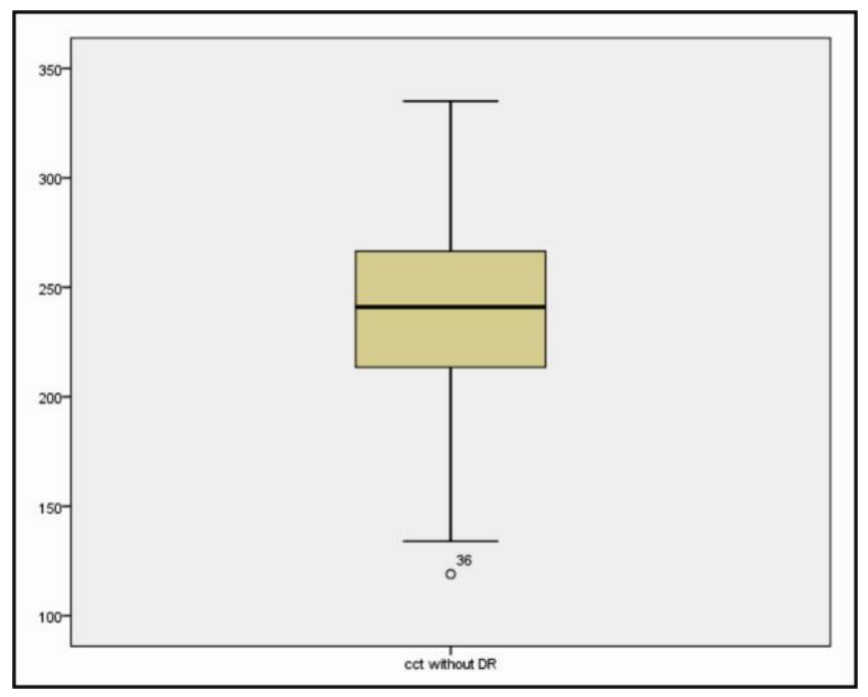

Figure 2c: Normality plot of central choroidal thickness in diabetic patients without diabetic retinopathy (Group B).

increase in sub-foveal choroidal thickness. Based on their findings they made the observation that retinal vasculature changes affected the choroidal layer. ${ }^{11}$ Reliable and reproducible choroidal layer thickness measurements have been made possible through the advent of spectral domain OCT. ${ }^{12}$ Our result did not show any relation of diabetic retinopathy with central Choroidal thickness. However, studies have shown that metabolic changes in diabetic patients affect the vasculature of choroid and may thus play a role in development of diabetic retinopathy. ${ }^{14}$ In one study it was seen that CCT was significantly thicker in severe diabetic retinopathy as compared to the mild disease. ${ }^{15}$ 
Another researcher showed that Choroidal thickness was altered in diabetes and was related to the severity of retinopathy. He also described the association of diabetic macular edema with significant decrease in the choroidal thickness. ${ }^{16}$ Similar results were shown by other authors. ${ }^{17}$

Contrary to the above mentioned studies, our results were consistent with a study which indicated no difference in the choroidal volume of patients with PDR and control subject. ${ }^{18}$ It was recently reported that CCT increased in the early stage of DR, and further decreased with DR progression. ${ }^{19}$ Yet another study has shown that disease duration was associated with a reduction of choroidal thickness and decreased CCT proved to be correlated with the severity of DR. ${ }^{20}$ The authors explained that for outer retinal layers, choroidal vasculature is the main source of nutrition. Any change affecting the choroid can indirectly affect the metabolic state and functioning of retina.

Strength of this study is that choroidal thickness was measured at a higher resolution and with an accurate device by adjusting various confounding factors. There are certain limitations of our study. It was a cross sectional study. A longitudinal study may be carried out to document the changes occurring over a period of time in diabetic patients. We also did not compare the severity of retinopathy with choroidal thickness. The study was limited to patients with type 2 diabetes only. The scope of the study can be further enhanced with inclusion of type-1 diabetics.

\section{CONCLUSION}

There is no significant difference in the central choroidal thickness between the diabetic patients with diabetic retinopathy and the patients without diabetic retinopathy.

\section{Ethical Approval}

The study was approved by the Institutional review board/ Ethical review board. (EC Ref No: 01/19)

\section{Conflict of Interest}

Authors declared no conflict of interest.

\section{REFERENCES}

1. Adhi M, Brewer E, Waheed N, Duker J. Analysis of morphological features and vascular layers of choroid in diabetic retinopathy using spectral-domain optical coherence tomography. JAMA Ophthalmol. 2013; 131 (10): $1267-1274$.

Doi: 10.1001/jamaophthalmol.2013.4321.

2. Sim D, Keane P, Mehta H, Fung S, Zarranz-Ventura J, Fruttiger M, et al. Repeatability and reproducibility of choroidal vessel layer measurements in diabetic retinopathy using enhanced depth optical coherence tomography. Invest Ophthalmol Vis Sci. 2013; 54 (4): 2893-2901. Doi: 10.1167/iovs.12-11085.

3. Spaide R, Koizumi H, Pozzoni M. Enhanced depth imaging spectral-domain optical coherence tomography. Am J Ophthalmol. 2008; 146 (4): 496500 .

4. Xu J, Xu L, Du K, Shao L, Chen C, Zhou J, et al. Subfoveal choroidal thickness in diabetes and diabetic retinopathy. Ophthalmology, 2013; 120 (10): 20232028. Doi: 10.1016/j.ophtha.2013.03.009.

5. Lee H, Lim J, Shin M. Comparison of choroidal thickness in patients with diabetes by spectral-domain optical coherence tomography. Korean J Ophthalmol. 2013; 27 (6): 433-439. Doi:10.3341/kjo.2013.27.6.433.

6. Regatieri C, Branchini L, Carmody J, Fujimoto J, Duker J. Choroidal thickness in patients with diabetic retinopathy analyzed by spectral-domain optical coherence tomography. Retina, 2012; 32 (3): 563-568. Doi: 10.1097/IAE.0b013e31822f5678.

7. Querques G, Lattanzio R, Querques L, Del Turco C, Forte R, Pierro L, et al. Enhanced depth imaging optical coherence tomography in type 2 diabetes. Invest Ophthalmol Vis Sci. 2012; 53 (10): 6017-6024.

Doi: 10.1167/iovs.12-9692.

8. Vujosevic S, Martini F, Cavarzeran F, Pilotto E, Midena E. Macular and peripapillary choroidal thickness in diabetic patients. Retina, 2012; 32 (9): 1781-1790. Doi: 10.1097/IAE.0b013e31825db73d.

9. Ferreire JT, Vicente A, Proenca R, Santos B, Cunha J, Alves M. Choroidal thickness in diabetic patients without diabetic retinopathy. Retina, 2018; 38 (4): 795804. Doi: 10.1097/IAE.0000000000001582.

10. Sudhalkar A, Chnablani KJ, Venkata A, Raman R, Rao S, Jonnadula BG. Choroidal thickness in diabetic patients of Indian ethnicity. Indian Journal of Ophthalmology, 2015; 63 (12): 912-916.

Doi: 10.4103/0301-4738.176024.

11. Ferrar D, Waheed NK, Duker JS. Investigating the choriocapillaries and choroidal vasculature with new optical coherence tomography technologies. Prog Retina Eye, 2015; 52: 130-155.

Doi:10.1016/j.Preteyers.2015.10.002. 
12. Rayess N, Rahimy E, Ying GS, Bagheri N, Ho AC, Regillo CD, et al. Baseline choroidal thickness as a predictor for response to anti-vascular growth factor therapy in diabetic macular edema. Am J Ophthalmol. 2015; 159 (1): 85-91. Doi:10.1016/jj.ajo.2014.09.033.

13. Abidia B, Sunen I, Calvo P, Bartol F, Verdes G, Ferreras A. Choroidal thickness measured using swept-source optical coherence tomography is reduced in patients with type 2 diabetes. Plos one, 2018; 13 (2). Doi: 10.1371/journal.pone.0191977.

14. Okamoto M, Matsuura T, Ogata N. Effects of panretinal photocoagulation on choroidal thickness and choroidal blood flow in patients with severe nonproliferative diabetic retinopathy. Retina, 2016; 36: 805-811. Doi: 10.1097/IAE.0000000000000800.

15. Ohara Z, Tabuchi H, Nakakura S, Yoshizumi Y, Sumino H, Maeda Y, et al. Changes in choroidal thickness in patients with diabetic retinopathy. Int Ophthalmol. 2018; 38 (1): 279-286.

Doi: 10.1007/s10792-017-0459-9.

16. Regatieri CV, Branchini L, Carmody J, Fujimoto JG, Duker JS. Choroidal thickness in patients with diabetic retinopathy analyzed by spectral-domain optical coherence tomography. Retina, 2012; 32: 563568. Doi: 10.1097/IAE.0B013E31822F5678.

17. Unsal E, Eltutar K, Zirtiloğlu S, Dinçer N, Ozdoğan Erkul S, Güngel H. Choroidal thickness in patients with diabetic retinopathy. Clin Ophthalmol. 2014; 8: 637-642. Doi: 10.2147/OPTH.S59395
18. Schocket LS, Brucker AJ, Niknam RM, Grunwald JE, DuPont J, Brucker AJ. Foveolar choroidal hemodynamics in proliferative diabetic retinopathy. Int Ophthalmol. 2004; 25 (2): 89-94.

Doi: $10.1023 / \mathrm{b}:$ inte.0000031744.93778.60.

19. Wang W, Liu S, Qiu Z, He M, Wang L, Li Y, Huang W. Choroidal Thickness in Diabetes and Diabetic Retinopathy: A Swept Source OCT Study. Invest Ophthalmol Vis Sci. 2020; 61 (4): 29.

Doi: 10.1167/iovs.61.4.29.

20. Horváth H, Kovács I, Sándor GL, Czakó C, Mallár $\mathbf{K}$, Récsán $\mathbf{Z}$, et al. Choroidal thickness changes in non-treated eyes of patients with diabetes: swept-source optical coherence tomography study. Acta Diabetol. 2018; 55 (9): 927-934.

Doi: $10.1007 / \mathrm{s} 00592-018-1169-0$

\section{Authors' Designation and Contribution}

Muhammad Ali Haider; Assistant Professor: Concepts, Literature search, Data acquisition, Manuscript preparation, Manuscript editing, Manuscript review.

Uzma Sattar; Investigative Oculist: Design, Data analysis, Statistical analysis, Manuscript review.

Muhammad Amjad; Consultant Ophthalmologist: Literature search, Manuscript editing, Manuscript review. 